PROCEEDINGS OF THE

AMERICAN MATHEMATICAL SOCIETY

Volume 125, Number 5, May 1997, Pages 1443-1450

S $0002-9939(97) 03742-8$

\title{
ON THE BOCHNER-RIESZ MEANS OF CRITICAL ORDER
}

\author{
E. R. LIFLYAND
}

(Communicated by J. Marshall Ash)

\begin{abstract}
Stein's well-known logarithmic asymptotics of the Lebesgue constants of the Bochner-Riesz means of critical order is extended to Lebesgue constants of more general linear means of multiple Fourier series. These means are generated by certain class of functions supported in convex domains with boundaries of non-vanishing Gaussian curvature.
\end{abstract}

\section{INTRODUCTION}

Let $f$ be an integrable function on $\mathbb{T}^{n}=(-\pi, \pi]^{n}, 2 \pi$-periodic in each variable. Let $f$ have a multiple Fourier series

$$
\sum_{k} \hat{f}(k) e^{i k \cdot x}
$$

where $x=\left(x_{1}, \ldots, x_{n}\right)$ is a point in the real $n$-dimensional Euclidean space $\mathbb{R}^{n}$, $k=\left(k_{1}, \ldots, k_{n}\right) \in \mathbb{Z}^{n}$ - the lattice of points in $\mathbb{R}^{n}$ with integer coordinates, $k \cdot x=$ $k_{1} x_{1}+\ldots+k_{n} x_{n}$ is the scalar product, and

$$
\hat{f}(k)=(2 \pi)^{-n} \int_{\mathbb{T}^{n}} f(x) e^{-i k \cdot x} d x
$$

is the $k$-th Fourier coefficient of function $f$.

Consider a sequence of linear operators

$$
L_{N}^{\lambda}: f \mapsto L_{N}^{\lambda}(f ; x)=\sum_{k} \lambda\left(\frac{k}{N}\right) \hat{f}(k) e^{i k \cdot x},
$$

where $\lambda$ is a continuous function. The operators map $C\left(\mathbb{T}^{n}\right)$ into $C\left(\mathbb{T}^{n}\right)$, or $L_{1}\left(\mathbb{T}^{n}\right)$ into $L_{1}\left(\mathbb{T}^{n}\right)$. It is well known that the norms in both cases are the same. They are called the Lebesgue constants. When $\lambda$ has a compact support, say $S$, the norms can be written explicitly

$$
\left\|L_{N}^{\lambda}\right\|=(2 \pi)^{-n} \int_{\mathbb{T}^{n}}\left|\sum_{k} \lambda\left(\frac{k}{N}\right) e^{i k \cdot x}\right| d x .
$$

The summation is taken over all $k \in N S \cap \mathbb{Z}^{n}$, where $N S=\{y: y=N x, x \in S\}$.

Received by the editors January 4, 1994 and, in revised form, November 27, 1995.

1991 Mathematics Subject Classification. Primary 42A24.

Key words and phrases. Lebesgue constants, Bochner-Riesz means, critical order.

The author acknowledges the support of the Minerva Foundation in Germany through the Emmy Noether Institute in Bar-Ilan University.

(c)1997 American Mathematical Society 
The most famous case is represented by the Bochner-Riesz means $(\lambda(x)=$ $\left.R_{\alpha}(x)=\left(1-|x|^{2}\right)_{+}^{\alpha}, \alpha \geq 0\right)$, see [Bc]. The value $\frac{n-1}{2}$ is called critical. Stein's well-known result $[\mathrm{S}]$ says that

$$
\left\|L_{N}^{R_{N-1}}\right\|=\omega_{n} \ln N+o(\ln N)
$$

where $\omega_{n}$ is some positive constant depending only on $n$ (not indicated explicitly in $[\mathrm{S}]$ ). This asymptotics makes it clear why it is often said that the Bochner-Riesz means of critical order behave as one-dimensional partial sums. In [Be] and [BL] this result was obtained as a simple corollary of certain (different) rather general theorems.

Our main goal is to generalize this result to a wider class of functions $\lambda$ (in connection with their supports). Let $S$ be the compact support of a function $\lambda$, with the boundary $\partial S$. Let $\lambda(x)=\lambda_{p, \alpha}(x)$ be $p$-smooth inside $S$, and in a certain neighborhood of $\partial S$ may be represented as follows:

$$
\lambda(x)=\lambda_{p, \alpha}(x)=f(x)(\rho(x))^{\alpha},
$$

where $f \in C^{p}\left(\mathbb{R}^{n}\right)$ and does not vanish on $\partial S, \rho(x)=0$ if $x \notin S, \rho(x)=\operatorname{dist}(x, \partial S)$ if $x \in S$. Notice, that $\rho$ is a smooth function in a neighborhood of $\partial S$ (see e.g., [Gi, Appendix B]).

Throughout the text we denote by $C, C_{1}$ some absolute positive constants, possibly different even if the same notation appears at one line.

\section{BASIC RESULTS}

Theorem. Let $S$ be the compact support of a function $\lambda=\lambda_{n, \frac{n-1}{2}}$, with the $n$ smooth boundary $\partial S$. Assume that $S$ is convex and the principal curvatures of $\partial S$ never vanish. Then there exists a positive constant $C_{S, \lambda}$ depending only on $S$ and $\lambda$ such that

$$
\left\|L_{N}^{\lambda}\right\|=C_{S, \lambda} \ln N+o(\ln N)
$$

for large $N$.

The proof relies on two basic facts. The following theorem, which is a part of Belinskii's result [Be, Theorem 1], gives the first basic fact.

Let $\Delta_{z}^{m}\left(\lambda ; h_{1}, \ldots, h_{m}\right)$ be the $m$-th difference of a function $\lambda$ defined recursively by the formulas

$$
\begin{aligned}
\Delta_{z}^{1}\left(\lambda ; h_{1}\right) & =\lambda\left(z+h_{1}\right)-\lambda(z) ; \\
\Delta_{z}^{m}\left(\lambda ; h_{1}, \ldots, h_{m}\right) & =\Delta_{z+h_{m}}^{m-1}\left(\lambda ; h_{1}, \ldots, h_{m-1}\right)-\Delta_{z}^{m-1}\left(\lambda ; h_{1}, \ldots, h_{m-1}\right),
\end{aligned}
$$

where $h_{j}, z \in \mathbb{R}^{n}, m$ is an arbitrary integer. Let

$$
\hat{\lambda}(x)=\int_{\mathbb{R}^{n}} \lambda(u) e^{i u x} d u
$$

be the Fourier transform of any integrable function $\lambda$. 
Theorem A. For each boundedly supported continuous function $\lambda$

$$
\begin{aligned}
\left\|L_{N}^{\lambda}\right\| & =(2 \pi)^{-n} \int_{N \mathbb{T}^{n}}\left|\prod_{j=1}^{n} \frac{x_{j}}{2 N \sin \frac{x_{j}}{2 N}} \hat{\lambda}(x)\right| d x+O\left\{\sum_{j=1}^{m-1} \int_{N \mathbb{T}^{n}}\left|\frac{x}{N}\right|^{j}|\hat{\lambda}(x)| d x\right. \\
& \left.+\int_{\frac{1}{2 \pi} \mathbb{T}^{n}} \ldots \int_{\frac{1}{2 \pi} \mathbb{T}^{n}}\left[\sum_{k}\left|\Delta_{\frac{k}{N}}^{m}\left(\lambda ; \frac{u_{1}}{N}, \ldots, \frac{u_{m}}{N}\right)\right|^{2}\right]^{\frac{1}{2}} d u_{1} \ldots d u_{m}\right\} .
\end{aligned}
$$

The proof of this result may be found also in Appendix to the paper [LRZ].

In order to apply Theorem A, we need some information about the behavior of the Fourier transform. The asymptotics of the Fourier transform of functions of the type specified is our second basic fact. This result is a consequence of a theorem describing the singularities of the Radon transform of such functions (see [RZ1], [RZ2]) and may be found in [LRZ] (see also [RZ3]).

Theorem B. Let $S$ be the compact support of a function $\lambda=\lambda_{p, \alpha}$ with $\alpha \geq 0$ and $p>\max \left(1, \frac{n-1}{2}+\alpha\right)$. Let $S$ be convex, with the $p$-smooth boundary $\partial S$, and suppose the principal curvatures of $\partial S$ never vanish. Let $\theta \in \mathbb{R}^{n}$ be a vector on the unit sphere, $x^{+}(\theta)$ and $x^{-}(\theta)$ be the (uniquely defined) points of $\partial S$ at which the function $\theta_{1} x_{1}+\cdots+\theta_{n} x_{n}$ attains maximum and minimum on $\partial S$, respectively. Then for $t \rightarrow+\infty$

$$
\begin{aligned}
\hat{\lambda}\left(t \theta_{1}, \ldots, t \theta_{n}\right) & =t^{-\alpha-\frac{n+1}{2}}\left(\Xi^{+} e^{i t \theta x^{+}(\theta)}+\Xi^{-} e^{i t \theta x^{-}(\theta)}+o(1)\right), \\
\Xi^{ \pm} & =(2 \pi)^{\frac{n-1}{2}} \Gamma\left(\frac{n+1}{2}\right) \exp \left\{ \pm \pi i \frac{2 \alpha+n+1}{4}\right\} f\left(x^{ \pm}\right)\left(\varkappa^{ \pm}\right)^{-\frac{1}{2}},
\end{aligned}
$$

where the remainder term is small uniformly in $\theta$, and $\varkappa^{ \pm}$is the Gaussian curvature of $\partial S$ at the points $x^{ \pm}$, respectively.

\section{Proof of Theorem}

Let us use (2) with $m$ specified below, and obtain the asymptotics of the first term in the right-hand side of (2), and all the rest are estimated from above. Let us start with the estimates of the remainder terms in (2). As for the integrals from the sum in the first term of the remainder in (2), using spherical coordinates and applying Theorem B, we get

$$
\int_{0}^{C N} N^{-j} t^{j} t^{n-1} t^{-\frac{n+1}{2}-\frac{n-1}{2}} d t \leq C_{1} N^{-j} N^{\frac{n-1}{2}-\frac{n-1}{2}+j}=O(1)
$$

for each $j=1, \ldots, m-1$. Now consider the last term in (2). Our computations are similar to those used by Belinskii for the usual Bochner-Riesz means [Be] and to those fulfilled in general situation in [LRZ] (see also [CoS]). It suffices to estimate 
the larger quantity

$$
\begin{aligned}
\sup _{u_{1}, \ldots, u_{m} \in \frac{1}{2 \pi} \mathbb{T}^{n}} & \left\{\sum_{k}\left|\Delta_{\frac{k}{N}}^{m}\left(\lambda ; \frac{u_{1}}{N}, \ldots, \frac{u_{m}}{N}\right)\right|^{2}\right\}^{\frac{1}{2}} \\
& =\sup _{u_{1}, \ldots, u_{m} \in \frac{1}{2 \pi} \mathbb{T}^{n}}\left\{\sum_{m+2 \leq \operatorname{dist}(k, \partial(N S)) \leq C N}\left|\Delta_{\frac{k}{N}}^{m}\left(\lambda ; \frac{u_{1}}{N}, \ldots, \frac{u_{m}}{N}\right)\right|^{2}\right. \\
& \left.+\sum_{0 \leq \operatorname{dist}(k, \partial(N S)) \leq m+2}\left|\Delta_{\frac{k}{N}}^{m}\left(\lambda ; \frac{u_{1}}{N}, \ldots, \frac{u_{m}}{N}\right)\right|^{2}\right\}^{\frac{1}{2}} .
\end{aligned}
$$

We may estimate from above each term in the last sum by the maximal value of $\lambda$ at the points with integer coordinates. This yields

$$
\sum_{0 \leq \operatorname{dist}(k, \partial(N S)) \leq m+2}\left|\Delta_{\frac{k}{N}}^{m}\left(\lambda ; \frac{u_{1}}{N}, \ldots, \frac{u_{m}}{N}\right)\right|^{2} \leq C \sum_{0 \leq \operatorname{dist}(k, \partial(N S)) \leq m+2}\left(\frac{m+2}{N}\right)^{2 \frac{n-1}{2}}
$$

The number of points with integer coordinates inside $N Y$, where $Y \subset \mathbb{R}^{n}$ is a convex domain, is equal to $N^{n} \operatorname{vol}(Y)+O\left(N^{n-1}\right)$, see e.g., [V]. Therefore, the last sum is not greater than

$$
C N^{-2 \frac{n-1}{2}} \sum_{0 \leq \operatorname{dist}(k, \partial(N S)) \leq m+2} 1 \leq C_{1} N^{n-1-2 \frac{n-1}{2}}=O(1) .
$$

Now consider the first sum on the right-hand side of (4). Use $m$ times the mean value theorem for the directional derivative. The sum being estimated is transformed to the form

$$
N^{-2 m} \sum_{m+2 \leq \operatorname{dist}(k, \partial(N S)) \leq C N}\left|\frac{\partial^{m} \lambda\left(\frac{k}{N}+\sigma_{1} \frac{u_{1}}{N}+\cdots+\sigma_{m} \frac{u_{m}}{N}\right)}{\partial u_{1} \ldots \partial u_{m}}\right|^{2} .
$$

Here $0<\sigma_{j}<1, j=1, \ldots, m$. We can take any $m$ such that $\frac{n+1}{2} \leq m \leq$ $n$. If $\frac{n-1}{2}$ is an integer the derivative is bounded, so this quantity is not greater than $C N^{n-2-2 \frac{n-1}{2}}=O(1)$. Otherwise, we estimate the derivative in the direction $\left(\sigma_{1}, \ldots, \sigma_{m}\right)$ by its maximal value, and obtain

$$
\begin{gathered}
N^{-2 m} \sum_{m+2 \leq \operatorname{dist}(k, \partial(N S)) \leq C N}\left|\frac{\partial^{m} \lambda\left(\frac{k}{N}+\sigma_{1} \frac{u_{1}}{N}+\cdots+\sigma_{m} \frac{u_{m}}{N}\right)}{\partial u_{1} \ldots \partial u_{m}}\right|^{2} \leq \\
C_{1} N^{-2 m} \sum_{m+2 \leq \operatorname{dist}(k, \partial(N S)) \leq C N}\left[\operatorname{dist}\left(\frac{k+\sigma_{m, k}}{N}, \partial S\right)\right]^{2\left(\frac{n-1}{2}-m\right)},
\end{gathered}
$$


where $0 \leq \sigma_{m, k} \leq m$. Let us denote $k+\sigma_{m, k}$ by $\bar{k}$. We have provided $m>\frac{n-1}{2}$ :

$$
\begin{aligned}
& N^{-2 m} \sum_{\frac{m+2}{N} \leq \operatorname{dist}\left(\frac{k}{N}, \partial S\right) \leq C}\left[\operatorname{dist}\left(\frac{\bar{k}}{N}, \partial S\right)\right]^{2\left(\frac{n-1}{2}-m\right)} \\
= & N^{-2 m} \sum_{m+2 \leq q \leq C N} \sum_{\frac{q}{N} \leq \operatorname{dist}\left(\frac{k}{N}, \partial S\right) \leq \frac{q+1}{N}}\left[\operatorname{dist}\left(\frac{\bar{k}}{N}, \partial S\right)\right]^{n-1-2 m} \\
\leq & C N^{-2 m} \sum_{1 \leq q \leq C N} \sum_{\frac{q}{N} \leq \operatorname{dist}\left(\frac{k}{N}, \partial S\right) \leq \frac{q+1}{N}}\left(\frac{q}{N}\right)^{n-1-2 m} \\
= & C N^{-n+1} \sum_{1 \leq q \leq C N} q^{n-1-2 m} \sum_{\substack{\frac{q}{N} \leq \operatorname{dist}\left(\frac{k}{N}, \partial S\right) \leq \frac{q+1}{N} \\
\leq}} N^{-n+1} N^{n-1} \sum_{1 \leq q \leq C N} q^{n-1-2 m} \leq C\left(1+N^{n-1-2 m+1}\right) \\
\leq & C\left(1+N^{n-2 \frac{n+1}{2}}\right)=O(1) .
\end{aligned}
$$

Collecting (3)-(7), we obtain that the remainder in (2) is bounded. Now we go on to the estimate of the main term. Let us pass to the spherical coordinates and apply Theorem B. Taking into account also that

$$
\frac{x_{j}}{2 N \sin \frac{x_{j}}{2 N}}-1=O\left(\frac{x_{j}^{2}}{N^{2}}\right)
$$

which leads to estimates like the ones for the remainder terms (see (6)), we get the following in place of the main term:

$$
(2 \pi)^{-n}(2 \pi)^{\frac{n-1}{2}} \Gamma\left(\frac{n+1}{2}\right) \int_{|\theta|=1} d \theta \int_{1}^{\pi N}\left|\Xi^{+}(\theta) e^{i t x^{+}(\theta) \theta}+\Xi^{-}(\theta) e^{i t x^{-}(\theta) \theta}\right| \frac{d t}{t}+o(\ln N) .
$$

Consider the inner integral in an equivalent form

$$
\int_{1}^{\pi N}\left|\Xi^{+}(\theta) e^{i t\left(x^{+}(\theta)-x^{-}(\theta)\right) \theta}+\Xi^{-}(\theta)\right| \frac{d t}{t}
$$

and estimate it, denoting $\left(x^{+}(\theta)-x^{-}(\theta)\right) \theta$ by $d(\theta)$. The following relation is very well-known (see e.g., [Z, Vol.1, Ch.2]):

$$
\int_{1}^{\pi N}\left|\frac{\sin t}{t}\right| d t=\frac{2}{\pi} \ln N+O(1)
$$

Let us adopt the method used in $[\mathrm{Z}]$ to get (8) to our conditions. The integral under estimation is equal to

$$
\int_{d(\theta)}^{\pi N d(\theta)}\left|\Xi^{+}(\theta) e^{i t}+\Xi^{-}(\theta)\right| \frac{d t}{t}=\sum_{k=1}^{[N d(\theta)]} \int_{2 k \pi}^{2(k+1) \pi}\left|\Xi^{+}(\theta) e^{i t}+\Xi^{-}(\theta)\right| \frac{d t}{t}+O(1) .
$$


The integral on the right-hand side is equal to

$$
\begin{aligned}
& \sum_{k=1}^{[N d(\theta)]} \int_{\frac{2 k \pi}{N d(\theta)}}^{\frac{2(k+1) \pi}{N d(\theta)}}\left|\Xi^{+}(\theta) e^{i t N d(\theta)}+\Xi^{-}(\theta)\right| \frac{d t}{t} \\
& =\int_{0}^{\frac{2 \pi}{N d(\theta)}}\left|\Xi^{+}(\theta) e^{i t N d(\theta)}+\Xi^{-}(\theta)\right| \sum_{k=1}^{[N d(\theta)]} \frac{1}{t+\frac{2 k \pi}{N d(\theta)}} d t .
\end{aligned}
$$

The last sum is equal to $\frac{N d(\theta)}{2 \pi}[\ln N d(\theta)+O(1)]$. Thus, we need to estimate

$$
\begin{aligned}
& \frac{1}{2 \pi}(2 \pi)^{-\frac{n+1}{2}} \Gamma\left(\frac{n+1}{2}\right) \int_{|\theta|=1} N d(\theta)[\ln N d(\theta)+O(1)] d \theta \\
& \cdot \int_{0}^{\frac{2 \pi}{N d(\theta)}}\left|\Xi^{+}(\theta) e^{i t N d(\theta)}+\Xi^{-}(\theta)\right| d t \\
& =(2 \pi)^{-\frac{n+3}{2}} \Gamma\left(\frac{n+1}{2}\right) \int_{|\theta|=1}[\ln N d(\theta)+O(1)] d \theta \int_{0}^{2 \pi}\left|\Xi^{+}(\theta) e^{i t}+\Xi^{-}(\theta)\right| d t \\
& =\ln N(2 \pi)^{-\frac{n+3}{2}} \Gamma\left(\frac{n+1}{2}\right) \int_{|\theta|=1} d \theta \int_{0}^{2 \pi}\left|\Xi^{+}(\theta) e^{i t}+\Xi^{-}(\theta)\right| d t+O(1) .
\end{aligned}
$$

Denoting $f\left(x^{ \pm}(\theta)\right)\left(\varkappa^{ \pm}(\theta)\right)^{-\frac{1}{2}}$ by $\phi^{ \pm}(\theta)$ (it should be recalled that $f$ and the Gaussian curvature $\varkappa$ do not vanish anywhere) we have to estimate

$$
\begin{aligned}
& (2 \pi)^{-\frac{n+3}{2}} \Gamma\left(\frac{n+1}{2}\right) \int_{|\theta|=1} d \theta \int_{0}^{2 \pi}\left|\phi^{+}(\theta) e^{\frac{i \pi n}{2}} e^{i t}+\phi^{-}(\theta) e^{-\frac{i \pi n}{2}}\right| d t \\
& =(2 \pi)^{-\frac{n+3}{2}} \Gamma\left(\frac{n+1}{2}\right) \int_{|\theta|=1} d \theta \int_{0}^{2 \pi}\left|(-1)^{n} \phi^{+}(\theta) e^{i t}+\phi^{-}(\theta)\right| d t .
\end{aligned}
$$

But the expression in the inner integral on the right-hand side of (9) never vanishes, and the right-hand side of (9) may be denoted by $C_{S, \lambda}$. The proof is complete.

Remark 1. Let us find $C_{S, \lambda}$ for usual Bochner-Riesz means of critical order; that is, let us find $\omega_{n}$ in (1). Here $S$ is the unit sphere, so $\varkappa=1$ everywhere. Since $\lambda(x)=\left(1-|x|^{2}\right)_{+}^{\frac{n-1}{2}}$, the function $f(x)=(1+|x|)^{\frac{n-1}{2}}$. On the boundary, that is, for $|x|=1$, we get $f\left(x^{ \pm}(\theta)\right)=2^{\frac{n-1}{2}}$. Taking into account the following well-known identity

$$
\int_{|\theta|=1} d \theta=\frac{2 \pi^{\frac{n}{2}}}{\Gamma\left(\frac{n}{2}\right)}
$$


we get

$$
\begin{aligned}
\omega_{n} & =(2 \pi)^{-n+3} \Gamma\left(\frac{n+1}{2}\right) \frac{2 \pi^{\frac{n}{2}}}{\Gamma\left(\frac{n}{2}\right)} 2^{\frac{n-1}{2}} \int_{0}^{2 \pi}\left|(-1)^{n} e^{i t}+1\right| d t \\
& =\frac{1}{2} \pi^{-\frac{3}{2}} \frac{\Gamma\left(\frac{n+1}{2}\right)}{\Gamma\left(\frac{n}{2}\right)} 8=\frac{4 \Gamma\left(\frac{n+1}{2}\right)}{\pi^{3 / 2} \Gamma\left(\frac{n}{2}\right)} .
\end{aligned}
$$

The same value was obtained in [BL] and, after correcting a small misprint, in [Be].

Remark 2. Generalizations of the Bochner-Riesz means of order $\alpha$ smaller than $\frac{n-1}{2}$ were considered earlier. Upper estimates of the Lebesgue constants were obtained in $[\mathrm{CoS}]$, and lower estimates in [LRZ], respectively. As in the spherical case (see [Ba], [IA]) the degree of growth of the Lebesgue constants is $N^{\frac{n-1}{2}-\alpha}$. For $\alpha>\frac{n-1}{2}$, the corresponding Lebesgue constants are bounded (see $[\mathrm{CoS}]$, it is also obvious in our case).

\section{ACKNOWLEDGEMENTS}

The author thanks A. Zaslavsky for several helpful discussions on the subject. The author also acknowledges the referee's remarks which definitely improved the presentation.

\section{REFERENCES}

[Ba] K. I. Babenko, On the Mean Convergence of Multiple Fourier Series and the Asymptotics of the Dirichlet Kernel of Spherical Means, Inst. Prikl. Mat. Akad. Nauk SSSR, Moscow, Preprint No. 52, 1971 (Russian).

[Bc] S. Bochner, Summation of multiple Fourier series by spherical means, Trans. Amer. Math. Soc. 40 (1936), $175-207$.

[Be] E. S. Belinskii, Behavior of Lebesgue constants for some methods of summation of multiple Fourier series, Metric Questions in the Theory of Functions and Mappings, Naukova Dumka, Kiev, 1977, pp. (19 - 39) (Russian). MR 58:29816

[BL] E. S. Belinskii, E. R. Liflyand, Lebesgue constants and integrability of Fourier transform of radial function, Dokl. of the Academy of Sciences of Ukraine (6) (1980), 5 - 10 (Russian). MR 81i:42016

[CoS] L. Colzani, P. M. Soardi, $L^{p}$ norms of certain kernels of the $N$-dimensional torus, Trans. Amer. Math. Soc 266 (2) (1981), 617 - 627. MR 84e:42008

[Gi] E. Giusti, Minimal surfaces and functions of bounded variation, Birkhäuser, Boston, 1984. MR 87a:58041

[IA] V. A. Ilyin, Sh. A. Alimov, Conditions for the convergence of expansions corresponding to self-adjoint extensions of elliptic operators. I. A self-adjoint extension of the Laplace operator with a point spectrum, Diff. Urav. 7 (1971), no. 4, 670-710 (Russian); English translation in Diff. Eq. 7 (1971), 615-650.

[LRZ] E. R. Liflyand, A. I. Zaslavsky, A. G. Ramm, Estimates from below for Lebesgue constants, J. of Fourier Analysis and Applications. 2 (1996), 287-301. CMP 96:09

[RZ1] A. G. Ramm, A. I. Zaslavsky, Singularities of the Radon transform, Bull. Amer. Math. Soc., 28 (1) (1993), 109 - 115. MR 93i:44003

[RZ2] - Reconstructing singularities of a function from its Radon transform, Math. Comp. Modelling 18 (1) (1993), 109-138. MR 94j:44006

[RZ3] _ Asymptotic behavior of the Fourier transform of piecewise-smooth functions, C. R. Acad. Sci. Paris 316 (6) (1993), 541-546. MR 94d:42019

[S] E. M. Stein, On certain exponential sums arising in multiple Fourier series, Ann. of Math. 73 (2) (1961), 87 - 109. MR 23:A2715 
[V] A. N. Varchenko, Number of lattice points in families of homothetic domains, Funk. Anal. i Ego Pril. 17 (1983), no, 2, 1-6 (Russian); English Translation in Funct. Anal. Appl. 17 (1983), 79-83. MR 85e:11077

[Z] A. Zygmund, Trigonometric series, Vol. I, II, Camb. Univ. Press, Cambridge, 1959. MR 21:6498

Department of Mathematics and Computer Science, Bar Ilan University, Ramat Gan, 52900, ISRAEL

E-mail address: liflyand@bimacs.cs.biu.ac.il 